\title{
Testing bronchodilator responsiveness
}

\author{
To the Editor:
}

The recent paper in the European Respiratory Journal by JANSON et al. [1] on testing bronchodilator responsiveness suggests that it has no value in distinguishing asthma from COPD. The authors correctly state that "there are many different ways of defining bronchodilator reversibility." However, they do not then mention any of them other than using the change in forced expiratory volume in $1 \mathrm{~s}\left(\mathrm{FEV}_{1}\right)$ standardised by the start value. This can lead to a sex and size bias in assessing bronchodilator response [2]. One method, that was recommended by the European Respiratory Society many years ago [3], standardises the change in $\mathrm{FEV}_{1}$ by the subject's predicted value and not their start value. Using this method it has been found that a change in $\mathrm{FEV}_{1}$ of $8 \%$ of predicted or more due to a bronchodilator was associated with a survival advantage [2]. This approach avoided all the pitfalls around the clinical diagnosis of COPD versus asthma. Previously it has been found that a change in $\mathrm{FEV}_{1}$ of $4 \%$ of predicted in COPD patients was associated with subjects being able to appreciate that their breathlessness was improved [4].

Before the respiratory community dismisses testing bronchodilator responsiveness based on the evidence of JANSON et al. [1], it needs to look at expressing any change due to a bronchodilator as a percent of the subject's predicted value, as this has been shown to be free from potential sex and size bias and is better at distinguishing important clinical end-points.

@ERSpublications

A paper in the ERJ suggests that testing bronchodilator responsiveness is not helpful but the authors have not used the most appropriate method http://bit.ly/2MJR3KM

Cite this article as: Miller MR. Testing bronchodilator responsiveness. Eur Respir J 2019; 54: 1902036 [https://doi.org/10.1183/13993003.02036-2019].

\author{
Martin R. Miller \\ Institute of Applied Health Sciences, University of Birmingham, Birmingham, UK.
}

Correspondence: Martin R. Miller, Institute of Applied Health Sciences, University of Birmingham, Birmingham, B15 2TT, UK. E-mail: martin@drmiller.uk

Received: 16 Oct 2019 | Accepted: 17 Oct 2019

Conflict of interest: M.R. Miller has nothing to disclose.

\section{References}

1 Janson C, Malinovschi A, Amaral AFS, et al. Bronchodilator reversibility in asthma and COPD: findings from three large population studies. Eur Respir J 2019; 54: 1900561.

2 Ward H, Cooper BG, Miller MR. Improved criterion for assessing lung function reversibility. Chest 2015; 148: 877-886.

3 Quanjer PH, Tammeling GJ, Cotes JE, et al. Official statement of the European Respiratory Society. Lung volumes and forced ventilatory flows. Report Working Party Standardization of Lung Function Tests, European Community for Steel and Coal. Eur Respir J 1993; 6: Suppl. 16, 5-40.

4 Redelmeier DA, Goldstein RS, Min ST, et al. Spirometry and dyspnea in patients with COPD. When small differences mean little. Chest 1996; 109: 1163-1168.

Copyright (CERS 2019

\section{From the authors:}

We thank M.R. Miller for his comments on our paper regarding bronchodilator reversibility in asthma and COPD [1]. We agree that it is important to look at different ways of defining bronchodilator reversibility. In our analysis, we investigated both flow-related bronchodilator reversibility, defined by the change in forced expiratory volume in $1 \mathrm{~s}\left(\mathrm{FEV}_{1}\right)$, and volume-related bronchodilator reversibility, defined 
by the change in forced vital capacity. We also looked at both the change in lung function parameters expressed as percent of the baseline value and the change in $\mathrm{FEV}_{1}$ standardised by the subject's predicted value. The latter was evaluated to control for the sex, age and height dependency of lung function. The results when reversibility was expressed as percent of the predicted value (in supplementary tables E3 and E4) [1] were the same as when reversibility was expressed as percent of the baseline value. Our interpretation was therefore that, in the present study, neither flow-related nor volume-related bronchodilator reversibility were independently associated with the symptom burden, health status or dyspnoea in the COPD population.

It should be noted that our study was population-based and thus it may better reflect real life conditions, such as those encountered in general practice. However, we agree that cohort studies on patients, which include a higher number of subjects with a severe COPD, could yield different results that may be more applicable to decision-making in specialist practice. Furthermore, as our analysis was cross-sectional, we could not assess a possible association between bronchodilator responsiveness and prognosis over time. We therefore agree with M.R. Miller that further studies are needed before the respiratory community can dismiss testing of bronchodilator responsiveness in COPD.

@ERSpublications

Neither flow-related nor volume-related bronchodilator reversibility were independently associated with the symptom burden, health status or dyspnoea in the COPD population http://bit.ly/2rigD1r

Cite this article as: Janson C, Malinovschi A, Amaral AFS, et al. Testing bronchodilator responsiveness. Eur Respir J 2019; 54: 1902104 [https://doi.org/10.1183/13993003.02104-2019].

Christer Janson $\circledast^{1,2}$, Andrei Malinovschi ${ }^{3}$, Andre F.S. Amaral $\odot^{2}$, Simone Accordini ${ }^{4}$, Jean Bousquet ${ }^{5,6,7}$, A. Sonia Buist ${ }^{8}$, Judith Garcia-Aymerich $\oplus^{9,10,11}$, Louisa Gnatiuc ${ }^{12}$, Wan $\operatorname{Tan}^{13}$, Kjell Torén ${ }^{14}$, Torsten Zuberbier and Peter Burney ${ }^{2}$ on behalf of all the authors

${ }^{1}$ Dept of Medical Sciences: Respiratory, Allergy and Sleep Research, Uppsala University, Uppsala, Sweden. ${ }^{2}$ Population Health and Occupational Disease, National Heart and Lung Institute, Imperial College London, London, UK. ${ }^{3}$ Dept of Medical Sciences: Clinical Physiology, Uppsala University, Uppsala, Sweden. ${ }^{4}$ Unit of Epidemiology and Medical Statistics, Dept of Diagnostics and Public Health, University of Verona, Verona, Italy. ${ }^{5}$ Fondation FMC VIA-LR, Montpellier, France. ${ }^{6}$ Euforea, Brussels, Belgium. ${ }^{7}$ Allergy Centre Charité, Dept of Dermatology and Allergy, Charité Universitätsmedizin Berlin, Berlin, Germany. ${ }^{8}$ Pulmonary and Critical Care Medicine, Oregon Health and Science University, Portland, OR, USA. ${ }^{9}$ ISGlobal, Barcelona, Spain. ${ }^{10}$ Universitat Pompeu Fabra (UPF), Barcelona, Spain. ${ }^{11}$ CIBER Epidemiología y Salud Pública (CIBERESP), Barcelona, Spain. ${ }^{12}$ Clinical Trial Service Unit and Epidemiological Studies Unit, Nuffield Dept of Population Health, University of Oxford, Oxford, UK. ${ }^{13}$ Centre for Heart Lung Innovation (Tan, Sin), University of British Columbia, St. Paul's Hospital Vancouver, Vancouver, BC, Canada. ${ }^{14}$ Dept of Occupational and Environmental Medicine, University of Gothenburg, Gothenburg, Sweden.

Correspondence: Christer Janson, Uppsala University, Dept of Respiratory Medicine, Akademiska sjukhuset, Uppsala, SE 751 85, Sweden. E-mail: christer.janson@medsci.uu.se

Received: 28 Oct 2019 | Accepted: 28 Oct 2019

Conflict of interest: C. Janson has nothing to disclose. A. Malinovschi has nothing to disclose. A.F.S. Amaral has nothing to disclose. S. Accordini has nothing to disclose. J. Bousquet reports personal fees and other funding from Chiesi, Cipla, Hikma, Menarini, Mundipharma, Mylan, Novartis, Sanofi-Aventis, Takeda, Teva and Uriach, and other funding from Kyomed, outside the submitted work. A.S. Buist has nothing to disclose. J. Garcia-Aymerich has nothing to disclose. L. Gnatiuc has nothing to disclose. W. Tan has nothing to disclose. K. Torén has nothing to disclose. T. Zuberbier has received consultancy fees from Bayer Health Care, FAES, Novartis and Henkel; has received grants/has grants pensing form Novartis and Henkel, and has received lecture fees from AstraZeneca, AbbVie, ALK, Almirall, Astellas, Bayer HealthCare, Bencradm Berlin Chemie, FAES, HAL, Leti, Meda, Menarini, Merck, MSD, Novartis, Pfizer, Sanofi, Stallergenes, Takeda, TEVA, UCB, Henkel, Kryolan and L'Oreal, outside the submitted work. P. Burney has nothing to disclose.

\section{Reference}

1 Janson C, Malinovschi A, Amaral AFS, et al. Bronchodilator reversibility in asthma and COPD: findings from three large population studies. Eur Respir J 2019; 54: 1900561. 